\title{
Primary Sternal Osteomyelitis Caused by Nocardia nova: Case Report and Literature Review
}

\author{
Ioannis G. Baraboutis ${ }^{1}$, Athena Argyropoulou ${ }^{2}$, Vasilios Papastamopoulos ${ }^{1}$, Zoi Psaroudaki ${ }^{2}$, \\ Olga Paniara ${ }^{2}$ and Athanasios T. Skoutelis ${ }^{1}$ \\ ${ }^{1}$ Infectious Diseases \& HIV Division, Department of Internal Medicine; ${ }^{2}$ Department of Microbiology; Evaggelismos Athens General Hospital; \\ Athens, Greece
}

\begin{abstract}
A 51 year old woman without significant past medical history or risk factors for Nocardia infection developed primary Nocardia nova sternal osteomyelitis with mediastinal abscess, diagnosed with open biopsy. She required prolonged antibiotic therapy and had a favorable outcome. Primary sternal osteomyelitis develops in the absence of a contiguous focus of infection, as opposed to secondary sternal osteomyelitis, which is usually a complication of sternotomy. Staphylococcus aureus probably still is the most common cause of both forms of sternal osteomyelitis. Nocardia species invade humans usually through the respiratory tract and can cause a variety of localized infections through the hematogenous route. Pulmonary involvement may or may not coexist. Immunosuppressed patients are more prone to infection by Nocardia species, although cases involving seemingly immunocompetent patients are not rare. This is the first reported case in the English literature of primary sternal osteomyelitis due to Nocardia nova or any other Nocardia species.
\end{abstract}

Key-Words: Nocardia nova, primary sternal osteomyelitis, secondary sternal osteomyelitis, Nocardia osteomyelitis.

Primary sternal osteomyelitis is a rare syndrome, with Staphylococcus aureus being the most frequent offender. Nocardia species can cause a variety of infectious syndromes, of which osteomyelitis occupies a relatively small percentage. When a relatively rare syndrome is caused by a pathogen that is not high on the differential diagnosis list, the result can be a challenging and fascinating case.

\section{Case Report}

A 51 year old Caucasian woman was admitted to the Rheumatology Department of our hospital due to a 3-week history of diffuse neck, shoulder and upper torso pain, exacerbated by movements. An outpatient trial of nonsteroidal anti-inflammatory medications had been unsuccessful. A few days later her pain was more localized above the manubrium, the area of the left clavicle and the left sternomastoid muscle, also fever was reported, up to $39.5^{\circ} \mathrm{C}$. The patient had no significant past medical history, was not using any medication and lived in a suburban area with her family. She was not working and she liked to do gardening in her spare time. There was no history of local trauma. On examination, there was intense redness, tenderness and swelling of the manubrium and the left sternoclavicular joint. Computed tomography (CT) of the chest revealed osteolytic changes of the manubrium and the presence of inflammatory tissue surrounding the manubrium and extending posteriorly. The lung parenchyma was unaffected. Brain and abdominal CT were unremarkable. A triple-phase bone scan was indicative of sternal osteomyelitis Received on 10 October 2007; revised 4 May 2008.

Address for correspondence: Dr Ioannis Baraboutis. Infectious Diseases \& HIV Division, Department of Internal Medicine, Evaggelismos Athens General Hospital. 45-47 Ipsilantou Street. Zip code: 10676. Athens, Greece. Phone: +30 210 7201242. Fax: + 30210 7201320. E-mail: ioannisbaraboutis@yahoo.gr.

The Brazilian Journal of Infectious Diseases 2008;12(3):257-259. (C) 2008 by The Brazilian Journal of Infectious Diseases and Contexto Publishing. All rights reserved. without any other bone involvement. Blood and urine cultures remained negative and the patient was empirically treated with high-dose intravenous vancomycin and ciprofloxacin with no response. Antibody testing to human immunodeficiency virus (HIV) and hepatitis B and C viruses were negative. An open biopsy was performed 1 week later, revealing persistent inflammatory tissue around the sternum and fluid collection posteriorly. Multiple bone specimens were sent for histoligical examination and culture. Histoligical changes were consistent with acute and chronic granulomatous inflammation. Gram-stain preparations of bone marrow and fluid revealed Grampositive beaded branched filaments, partially acid-fast with the modified Kinyoun's stain, suggesting Nocardia spp. Cultures of both types of specimens were carried out using standard microbiological techniques. After 72 hours of aerobic incubation, growth of chalky, white to orange-colored colonies, consistent with Nocardia spp., was observed. (Figure 1).

Further speciation to Nocardia nova complex was based on susceptibility testing performed by agar disk diffusion assay. The strain was susceptible to erythromycin and ampicillin and resistant to amoxicillin-clavulanic acid, exhibiting the type III drug pattern of the former $N$. asteroides complex [1,2].

Definite identification to the species level was done by broad-range 16s rRNA PCR followed by sequencing [3]. Analysis of the results by BLAST search demonstrated Nocardia nova (GenBank accession no. AB292584).

Susceptibility testing was performed by the E-test method (AB Biodisk, Solna, Sweden) and the strain was found to be sensitive to trimethoprim-sulfamethoxazole (TMP-SMX) with a MIC of $0.3 \mu \mathrm{g} / \mathrm{mL}$.

The patient was given high-dose intravenous TMP-SMX and had a favorable clinical response. The medication was continued on an outpatient basis and she completed 1 year of treatment without further sequelae. 
Figure 1. Colonies of N.nova on 7H10 Middlebrook agar (96 hours of incubation).

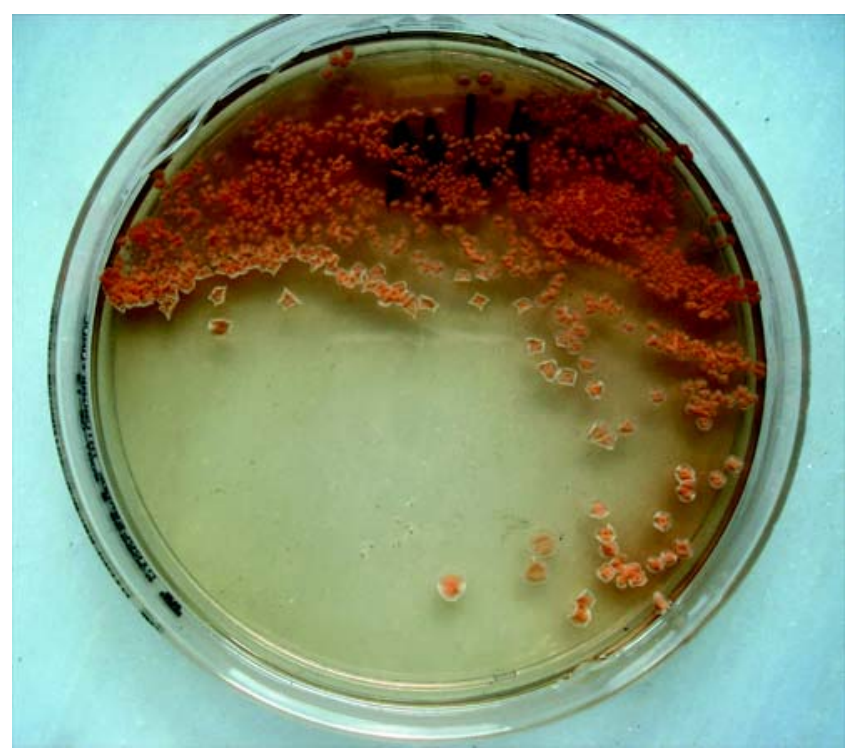

\section{Discussion}

Sternal osteomyelitis is a rare entity, comprising $0.3 \%$ of all cases of osteomyelitis in the literature [4]. Upon review of the English-language literature in 1989, Gill and Stevens found 57 reported cases [5]. Primary sternal osteomyelitis (PSO) has no contiguous focus of infection, whereas secondary sternal osteomyelitis (SSO) is usually a complication of sternotomy [6]. In the preantibiotic era, sternal osteomyelitis was often associated with staphylococcal bacteremia and sepsis, with a high associated mortality. Mortality associated with sternal osteomyelitis has decreased dramatically with the advent of antibiotics. In more recent decades, Staphylococcus aureus is still the predominant species responsible for both PSO and SSO, although Pseudomonas aeruginosa is predominant among intravenous drug abusers [7]. Salmonella, Klebsiella, Aspergillus, Mycobacterium tuberculosis and Actinomyces israelii have also been reported as causal organisms of PSO [4,8,9].

It is noteworthy that PSO is also rare in children. S. aureus and Salmonella (in sickle cell disease patients) are the commonest causes [10]. We were able to find, in the English literature, two pediatric PSO cases caused by communityacquired methicillin-resistant $S$. aureus. [10].

Reported risk factors for PSO include intravenous drug abuse with or without antecedent history of blunt trauma, acquired immunodeficiency syndrome, hemoglobinopathies and SAPHO syndrome (synovitis, acne, pustulosis, hyperostosis and osteomyelitis) [4,11]. In some cases, however, none of the above risk factors were identified.

PSO presents with anterior sternal pain, tenderness, redness, and swelling. It is often clinically subacute, unlike the clinically virulent SSO. Due to the rarity of the condition, diagnosis and appropriate treatment are often delayed. The differential diagnosis is broad and should include cellulitis, soft-tissue abscess, benign and malignant soft-tissue tumors, and bony tumors. Diagnosis requires a high index of suspicion and a needle or open bone biopsy. Plain roentgenograms and computed tomography are the central radiographic components in the workup [4]. Nuclear isotope scans, including technetium 99m and gallium 67, may also be useful [11].

Successful treatment of primary osteomyelitis has been reported with antibiotics alone on several occasions (for example, tuberculous PSO). Nevertheless, it is suggested, especially in the surgical literature, that early surgical treatment provides definitive treatment, with decreased morbidity and cost of care [4]. Medical management may be problematic in terms of a prolonged duration of antibiotic therapy, interference with work and other daily activities, and the possibility of treatment failure necessitating surgical debridement.

Nocardia was first described by Nocard in 1888. Nocardia species are aerobic bacteria that form branched filaments and can be found worldwide in soil, decaying vegetable matter and aquatic environments. They can become airborne, particularly on dust particles and usually are introduced as airborne spores or mycelial fragments through the respiratory tract. Nocardia asteroides is the predominant human pathogen.

Tsukamura first described $N$. nova in 1982 as a separate species, distinct from $N$. asteroides and $N$. farcinica [2,12]. In 1988, Wallace and colleagues reported six drug susceptibility patterns among a study of 78 clinical isolates previously identified as " $N$. asteroides" [1]. Detailed studies of isolates with the six individual $N$. asteroides drug patterns followed the initial 1988 report, and these new studies described several of the drug patterns as distinct species/taxa of Nocardia. Isolates with the type III drug susceptibility pattern were later shown to be a complex of organisms related to $N$. nova. $N$. nova complex organisms (including $N$. nova, $N$. veterana, $N$. africana, N. kruczakiae and maybe others) are susceptible or moderately susceptible to both amoxicillin and erythromycin and resistant to amoxicillin-clavulanic acid [12]. Although phenotypic methods and susceptibility patterns are used for identification, they are not as reliable as definitive identification methods. Gene sequencing (16s rRNA) provides a more definitive identification and is being increasingly used [12].

Nocardiosis is considered an opportunistic infection, although approximately one third of the infected patients are immunocompetent. It occurs, in particular, in patients with underlying malignancies, chronic lung disease (including alveolar proteinosis), or disorders of cell-mediated immunity, including HIV infection; those who receive long-term corticosteroid therapy; and those who undergo organ transplantation. Nocardiosis has also been associated with diabetes, alcoholism, tuberculosis, chronic granulomatous disease and treatment with tumor necrosis factor antagonists. It also occurs in intravenous drug abusers. The principal syndromes caused by Nocardia spp include upper and lower respiratory tract disease and hematogenous dissemination to the brain, skin and subcutaneous tissues, eyes (especially the retina), heart valves, liver, kidneys, joints and bones 
$[14,15]$. Extrapulmonary syndromes reported to be caused by $N$. nova complex organisms include bacteremia, catheterrelated bacteremia, aortitis, implantable defibrillator pocket infection, prosthetic hip joint infection, anterior scleritis, sinusitis, central nervous system infection, liver infection, primary cutaneous disease and disseminated disease [12].

Osteomyelitis is an unusual manifestation of infection with Nocardia spp. In an older review of 243 cases of Nocardia asteroides infections, there were no osteomyelitis cases, while a total of 12 cases of Nocardia spinal osteomyelitis have been published over the past 40 years [13,16]. Most cases of osteomyelitis are secondary, resulting from direct extension from a primary lung or cutaneous lesion.

There are also a limited number of reported cases of primary Nocardia osteomyelitis, due to hematogenous dissemination from pulmonary or soft tissue foci. Bones most frequently involved via the hematogenous route are the vertebral bodies, the femur and tibia. Less frequent locations are the ilium, fibula, metatarsal bones and mandible $[17,18]$. For detailed descriptions, the reader is referred to already published case- reports and reviews on primary Nocardia osteomyelitis $[17,18]$. Many of the reported patients with primary Nocardia osteomyelitis were immunocompetent like our patient, whose infection was most likely associated with her regular gardening activities. A lung lesion was or was not present and the adjacent soft tissues were secondarily involved in a large percentage of these patients. Prolonged antimicrobial therapy (at least 3 months), usually with trimethoprim-sulfamethoxazole, was the rule and most patients recovered. Our patient required an open biopsy of the chest to reach the diagnosis and almost 1 year of antibiotics.

We were able to find in the English literature only 2 cases of $N$. nova osteomyelitis $[13,19]$. The first one involved a 27 -yearold female renal transplant patient, who developed lumbar spine osteomyelitis with contiguous psoas abscess, necessitating open surgery, as a relapse-through the hematogenous routeof previous pulmonary nocardiosis. The second one involved a 29-year-old woman with primary cutaneous nocardiosis of the foot, involving the bones, successfully treated only with prolonged antibiotic therapy. Interestingly, both patients, like ours, were female, as opposed to the observed, in most case series, male preponderance in Nocardia infections.

To our knowledge, there is no previous reported case of PSO caused by Nocardia (any species). Regarding Nocardiainduced SSO, Wenger and colleagues reported in 1998 the first nosocomial $N$. farcinica sternotomy site infection outbreak (5 patients) following open heart surgery, which was linked to hand contamination of an anesthesiologist [20]. Another 3 cases of Nocardia-induced SSO ( 2 of them by $N$. asteroides) have been reported after heart valve replacement $[21,22]$.

In conclusion, this is the first reported case of primary sternal osteomyelitis due to Nocardia species. The possibility of nocardiosis needs to be included in the differential diagnosis of sternal osteomyelitis, even for apparently immunocompetent adults.

\section{Acknowledgements}

We thank Dr E. Petinaki, Department of Microbiology, University Of Thessaly, Larissa, Greece for molecular identification of the organism.

\section{References}

1. Wallace R.J., Jr., Steele L.C., Sumpter G., Smith J.M. Antimicrobial susceptibility patterns of Nocardia asteroides. Antimicrob Agents Chemother 1988;32(12):1776-9.

2. Wallace R.J., Jr., Brown B.A., Tsukamura M., et al. Clinical and laboratory features of Nocardia nova. J Clin Microbiol 1991;29:2407-11.

3. Gatselis N., Malli E., Papadamou G., et al. Direct Detection of Cardiobacterium hominis in Serum from a Patient with Infective Endocarditis by Broad-Range Bacterial PCR. J Clin Microbiol 2006;44(2):669-72.

4. Lin J.C., Miller S.R., Gazzaniga A.B. Primary sternal osteomyelitis. Ann Thorac Surg 1996;61(1):225-7.

5. Gill E.A., Stevens D.L. Primary sternal osteomyelitis. West J Med 1989;151(2):199-203.

6. Culliford A.T., Cunningham J.N., Jr., Ze R.H., et al. Sternal and costochondral infections following open-heart surgery-a review of 2594 cases. J Thorac Cardiovasc Surg 1976;72(5):714-26.

7. Boll K.L., Jurik A.G. Sternal osteomyelitis in drug addicts. J Bone Joint Surg Br 1990;72(2):328-9.

8. Pinilla I., Martin-Hervas C., Gil-Garay E. Primary sternal osteomyelitis caused by Actinomyces israelii. South Med J 2006;99(1):96-7.

9. Walker W.A., Pate J.W. Primary aspergillus osteomyelitis of the sternum. Ann Thorac Surg 1991;52(4):868-70.

10. Upadhyaya M., Keil A., Thonell S., et al. Primary sternal osteomyelitis: a case series and review of the literature. J Pediatr Surg 2005;40(10):1623-7.

11. Khoury J., Jerushalmi J., Kats I., et al. Imaging of primary sternal osteomyelitis in a patient with Crohn's disease. J Clin Imag 2003;27(5):358-62.

12. Brown-Elliott B.A., Brown J.M., Conville P.S., Wallace R.J., Jr. Clinical and Laboratory Features of the Nocardia spp. Based on Current Molecular Taxonomy. Clin Microbiol Rev 2006;19(2):259-82.

13. Nocardia nova as the causative agent in spondylodiscitis and psoas abscess. J Clin Microbiol 2007;45(1):262-5.

14. Watson A., French P., Wilson M. Nocardia Asteroides native valve endocarditis. Clin Infect Dis 2001;32(4):660-1.

15. Shin K.H., Lee W.S., Son Y.K., et al. Nocardia osteomyelitis in a pachymeningitis: an example of a difficult case to treat with antimicrobial agents. Yonsei Med J 1998;39(6):604-10.

16. Palmer D.L., Harvey R.L., Wheeler J.K. Diagnostic and therapeutic considerations in Nocardia asteroides infection. Medicine 1974;53:391-401.

17. Schwartz J.G., Tio F.O. Nocardial osteomyelitis: a case report and review of the literature. Diagn Microbiol Infect Dis. 1987;8(1):37-46.

18. Graat H.C., Van O.A., Day G.A., McPhee I.B. Nocardia farcinica spinal osteomyelitis. Spine 2002;27(10):E253-7.

19. Miller S.D. Madura foot: treatment of Nocardia nova infection with antibiotics alone. Am J Orthop 2001;30(6):495-8.

20. Wenger P.N., Brown J.M., McNeil M.M., Jarvis W.R. Nocardia farcinica sternotomy site infections in patients following open heart surgery. J Infect Dis 1998;178(5):1539-43.

21. Eigel P., Elert O., Hopp H., et al. Nocardial endocarditis after aortic valve replacement. Reports of two cases. Scand J Thorac Cardiovasc Surg 1988;22(3):289-90.

22. Yew W.W., Wong P.C., Kwan S.Y., et al. Two cases of Nocardia asteroides sternotomy infection treated with ofloxacin and a review of other active antimicrobial agents. J Infect 1991;23(3):297-302. 